\title{
Impact of Myocardial Scarring on Outcomes of Cardiac Resynchronization Therapy: Extent or Location?
}

\author{
Yi-Zhou Xu ${ }^{1}$, Yong-Mei Cha ${ }^{1-3}$, Dali Feng ${ }^{4}$, Brian D. Powell ${ }^{1}$, Heather J. Wiste ${ }^{5}$, Wei Hua ${ }^{3}$, \\ and Panithaya Chareonthaitawee ${ }^{1}$ \\ ${ }^{1}$ Division of Cardiovascular Diseases, Mayo Clinic, Rochester, Minnesota; ${ }^{2}$ Department of Cardiology, Hangzhou First People's \\ Hospital of Nanjing Medical University, Hangzhou, China; ${ }^{3}$ Cardiac Arrhythmia Center, Fu Wai Hospital of the Chinese Academy of \\ Medical Sciences and Peking Union Medical College, Beijing, China; ${ }^{4}$ Metropolitan Heart and Vascular Institute, Minneapolis, \\ Minnesota; and ${ }^{5}$ Division of Biomedical Statistics and Informatics, Mayo Clinic, Rochester, Minnesota
}

Refining the criteria for patient selection for cardiac resynchronization therapy (CRT) may improve its outcomes. The study objective was to determine the effect of scar location, scar burden, and left ventricular (LV) lead position on CRT outcomes. Methods: The study included 213 consecutive CRT recipients with radionuclide myocardial perfusion imaging before CRT between January 2002 and December 2008. Scar localization and myocardial viability were analyzed using a 17-segment model and a 5-point semiquantitative scale. New York Heart Association (NYHA) class and echocardiography were assessed before and after CRT. The anatomic LV lead location in the 17-segment model was assessed by review of fluoroscopic cinegrams in right and left anterior oblique views. As in published studies, clinical response was defined as an absolute improvement in LV ejection fraction of $\geq 5$ percentage points after CRT. Results: A total of 651 scar segments was identified in 213 patients. Eighty-three percent of scar segments were located in the LV anterior, posterior, septal, and apical regions, whereas $84 \%$ of LV leads were in the lateral wall. Only $11 \%$ of LV leads were positioned in scar segments. The extent of scarring was significantly higher in nonresponders than in responders $(18.0 \%$ vs. $6 \%, P=0.001)$. Compared with patients with scarring $>22 \%$, patients $\leq 70$ y with scarring $\leq 22 \%$ of the left ventricle had a greater increase in LV ejection fraction $(10.1 \% \pm$ $10.5 \%$ vs. $0.8 \% \pm 6.1 \% ; P<0.001)$ and improvement in NYHA class $(-0.9 \pm 0.7$ vs. $-0.5 \pm 0.8 ; P=0.02)$. Conclusion: LV leads were often located in viable myocardial regions. Less scar burden was associated with a greater improvement in heart failure but only in relatively younger CRT recipients.

Key Words: cardiac resynchronization therapy; heart failure; myocardial scarring; viability; imaging

J Nucl Med 2012; 53:47-54

DOI: 10.2967/jnumed.111.095448

\footnotetext{
Received Jul. 8, 2011; revision accepted Sep. 22, 2011.

For correspondence or reprints contact either of the following: Panithaya Chareonthaitawee, Division of Cardiovascular Diseases, Mayo Clinic, 200 First St. SW, Rochester, MN 55905.

E-mail: chareonthaitawee.panithaya@mayo.edu

Wei Hua, Fu Wai Hospital and Cardiovascular Institute, Chinese Academy of

Medical Sciences and Peking Union Medical College, Beijing, 100037, China.

E-mail: drhua@vip.sina.com

Published online Dec. 12, 2011.

COPYRIGHT @ 2012 by the Society of Nuclear Medicine, Inc.
}

$\mathbf{H}$ eart failure (HF) prevalence is estimated to be $1 \%-$ $2 \%$ in Western countries, with an incidence of approximately 5-10 per 1,000 persons per year. HF negatively affects quality of life and survival and accounts for $1 \%-$ $2 \%$ of all health-care expenditure in developed countries. Left ventricular (LV) systolic function underlies the traditional HF paradigm and, despite significant advances in treatment, 5-y mortality still approaches 50\% (1). Cardiac resynchronization therapy (CRT) is a treatment option for advanced HF despite optimal medical therapy, reduced LV ejection fraction (EF), and wide QRS complex. Randomized studies have demonstrated that CRT can improve cardiac function, clinical symptoms, quality of life, and even survival for patients with advanced HF (2-5). However, about one third of HF patients with reduced LVEF and wide QRS do not clinically respond after CRT. The reasons are multifactorial, and predicting who will respond to CRT remains a challenge $(2,6)$. The suboptimal selection of CRT candidates has been considered an important contributor to the less optimal response rate. Diverse strategies to identify cardiac mechanical dyssynchrony and other predictors of benefit from CRT have been developed to improve CRT patient selection $(7,8)$. Over 25 echocardiographic studies using LV dyssynchrony to predict CRT response have been published, reporting a sensitivity of 24\%$100 \%$ and specificity of $55 \%-100 \%$ (7). Careful analysis of this literature reveals many limitations, including small sample sizes, single-center studies, lack of consensus in CRT-response assessment, and variable LV dyssynchrony measurements. Similarly, cardiac MRI studies of LV dyssynchrony to predict CRT response have been reported but also with variable results and similar limitations. A promising predictor is the measurement of myocardial scarring, by either cardiac MRI or nuclear imaging; however, published studies also remain limited and inconclusive. Cardiac MRI studies suggest the role of scar location, scar burden, and scar transmurality in predicting CRT response, but most studies were small $(8)$. Nuclear studies report higher scar burden in CRT nonresponders than in responders but 
were limited similarly by their small sample size (9-12). Most studies did not assess lead position in relation to scar location. The purpose of this study was, therefore, to evaluate the relationships between myocardial scar distribution, location, and burden by nuclear imaging, LV lead position, and CRT clinical response and outcomes in a large cohort of patients with advanced $\mathrm{HF}$ and reduced LVEF.

\section{MATERIALS AND METHODS}

From January 1, 2002, through December 31, 2008, 720 consecutive patients with advanced HF received a CRT device at the Mayo Clinic. Of these, 213 patients had radionuclide myocardial perfusion imaging (MPI) within $1 \mathrm{y}$ before CRT and were included. All patients met standard CRT criteria: New York Heart Association (NYHA) functional class III or IV, LVEF $\leq$ $35 \%$, and QRS duration $\geq 120 \mathrm{~ms}$. Ischemic cardiomyopathy (ICM) was determined by coronary angiography as presence of coronary artery stenosis $\geq 70 \%$ in at least 1 major coronary artery or $\geq 50 \%$ in the left main (13). The study was approved by the Mayo Clinic Institutional Review Board, and all patients consented to participate in the study.

Baseline clinical characteristics were obtained from medical records. Each subject was evaluated for symptom status, HF etiology, NYHA functional class, and medication use. A 12-lead electrocardiogram was performed at baseline; QRS duration and morphology were assessed. The measurement of B-type natriuretic peptide (BNP) (14) was performed before and at 3 and 6 mo after CRT implantation.

Before CRT implantation, 46 patients underwent PET MPI, and 167 patients underwent SPECT MPI.

PET images were acquired with a PET Advance scanner (GE Healthcare) before CRT. After positioning in the scanner, a 4-min transmission scan was obtained to check optimal LV positioning in the field of view. A 10-min transmission scan was subsequently obtained for attenuation correction of the emission images. ${ }^{13} \mathrm{~N}$-ammonia (370-740 MBq [10-20 mCi]; $n=36$ ) or ${ }^{82} \mathrm{Rb}(1,850-2,220 \mathrm{MBq}$ [50-60 mCi]; $n=7)$ was injected intravenously at rest, followed immediately by the resting static emission acquisition.

SPECT images were acquired as described previously $(15,16)$ using either a 1 -d low-dose rest ${ }^{99 m}$ Tc-sestamibi protocol $(n=$ $153)$ or a delayed ${ }^{201} \mathrm{Tl}$ protocol $(n=17)$.

Only resting PET and resting or delayed SPECT myocardial perfusion images were analyzed for scarring in this study. The images were displayed in 3 orthogonal planes (short, horizontal long, and vertical long axes). Radioisotope uptake in each of the standard $17 \mathrm{LV}$ segments (17) was graded by a consensus of 2 experienced observers using a 5-point scoring system ( 0 , absent; 1 , severely decreased; 2 , moderately decreased; 3 , mildly decreased; and 4 , normal) (18). Scores of 0 and 1 were defined as indicating the presence of scarring, and scores of 2, 3, and 4 were defined as indicating viable myocardial tissue. These values were chosen because of prior demonstration that segments with relative tracer uptake $\geq 50 \%$ of normal myocardium are considered viable (19). Scar extent was calculated by dividing the number of scar segments by the total number of segments and expressed as \%LV. All images were interpreted by a consensus of 2 highly experienced nuclear cardiology readers who have adapted to interpreting nuclear MPI using different myocardial perfusion tracers with and without attenuation correction. This approach has been internally validated in our nuclear cardiology laboratory and was found to have a moderately high level of agreement.

Resting transthoracic 2-dimensional echocardiography was performed before and at a median of 6.8 mo (interquartile range [IQR], 4.1-11.3 mo) after CRT implantation. Standard long-axis 2- and 4-chamber images and calculation of LV end-systolic dimensions (LVESDs) and end-diastolic dimensions (LVEDDs) and LVEF were obtained using the Simpson biplane method. Mitral regurgitation (MR) severity was graded using standard techniques ( 0 , none or trivial; 1 , mild; 2 , moderate; and 3 , severe). Right ventricular enlargement and systolic dysfunction (for both, 0 , normal; 1 , mild; 2 , moderate; and 3 , severe) and pulmonary artery systolic pressure were estimated from the transtricuspid maximal regurgitant flow velocity.

Regarding CRT implantation and programming, commercially available leads and CRT devices were used. The lateral and posterolateral cardiac veins were the target of LV lead placement, as allowed by coronary venous anatomy, diaphragmatic stimulation, or pacing thresholds. The standard settings included DDD or DDDR mode and atrioventricular delay of $100 \mathrm{~ms}$ (sensed) and $130 \mathrm{~ms}$ (paced). The device was programmed in VVIR mode when chronic atrial fibrillation was present.

Six-month follow-up was recommended after device implantation but actual follow-up time varied, with a median time of 6.8 mo (IQR, 4.1-11.3 mo). NYHA status and echocardiography were reassessed. As in prior studies, clinical response was defined as an absolute improvement in LVEF of $\geq 5$ percentage points after CRT $(6,20)$. Survival status as of May 2009 was obtained using a national death and location database (Accurint; LexisNexis) (21). The median follow-up time was 3.2 y (IQR, 1.9-4.8 y).

The anatomic lead locations in the 17-segment model were assessed by review of fluoroscopic cinegrams in right and left anterior oblique views in available patients (22). The left ventricle was divided into 3 levels (basal, mid, and apical). To match the MPI segments, the basal and mid levels were each divided further into 6

TABLE 1

Baseline Demographics

\begin{tabular}{lc}
\hline \multicolumn{1}{c}{ Variable } & Overall value \\
\hline Age $(\mathrm{y})$ & $70.2 \pm 9.4$ \\
Male sex & $179(84)$ \\
NICM & $58(27)$ \\
Chronic atrial fibrillation & $58(27)$ \\
NYHA class & $3.0 \pm 0.5$ \\
QRS duration & $164.1 \pm 34.7$ \\
$\quad$ (before implantation) $(\mathrm{ms})$ & \\
Left bundle branch block & $99(49)$ \\
B-type natriuretic peptide & \\
$\quad$ (before implantation) $(\mathrm{pg} / \mathrm{mL})$ & \\
Median & 601.0 \\
IQR & $251.0-1,016.0$ \\
Angiotensin-converting & $172(82)$ \\
$\quad$ enzyme/angiotensin & \\
II receptor blocker inhibitor & \\
$\beta$-blocker & $186(89)$ \\
Aldactone & $64(30)$ \\
Digoxin & $118(56)$
\end{tabular}

Values are number, with percentage in parentheses, or mean \pm SD unless indicated otherwise. 
TABLE 2

Change in Variables (Before CRT vs. After CRT)

\begin{tabular}{|c|c|c|c|}
\hline Variable & Before CRT & After CRT & $P$ \\
\hline NYHA class & $3.0 \pm 0.4$ & $2.4 \pm 0.8$ & $<0.001$ \\
\hline QRS duration (ms) & $166.9 \pm 34.1$ & $162.5 \pm 24.5$ & 0.16 \\
\hline B-type natriuretic peptide (pg/mL) & & & 0.04 \\
\hline Median & 580.0 & 386.0 & \\
\hline IQR & $234.0-1,013.0$ & $164.5-863.5$ & \\
\hline LVEF (\%) & $23.8 \pm 7.3$ & $31.2 \pm 11.6$ & $<0.001$ \\
\hline LVEDD (mm) & $66.1 \pm 8.7$ & $63.6 \pm 9.6$ & $<0.001$ \\
\hline LVESD (mm) & $57.6 \pm 8.9$ & $53.7 \pm 10.8$ & $<0.001$ \\
\hline Right ventricular size, grade $0-3$ & $0.9 \pm 0.9$ & $0.8 \pm 0.9$ & 0.31 \\
\hline Right ventricular dysfunction, grade $0-3$ & $1.0 \pm 0.9$ & $0.9 \pm 0.9$ & 0.38 \\
\hline Left atrial size (mm) & $61.5 \pm 9.4$ & $62.4 \pm 9.3$ & 0.27 \\
\hline MR, grade $0-3$ & $1.5 \pm 0.8$ & $1.3 \pm 0.7$ & $<0.001$ \\
\hline
\end{tabular}

Values are mean \pm SD unless indicated otherwise.

segments, and the apex into 4 segments. The apex itself was also evaluated as a single segment to match the MPI segmental model. The fluoroscopic cinegrams were independently reviewed by 2 electrophysiologists. If a discrepancy was present, the cinegrams were reviewed again by the same 2 electrophysiologists together to reach a consensus. To evaluate the relation of scar segment and LV lead site, the data on LV lead position were merged with the 17-segment MPI model using a transparent overlay of the MPI and anatomic lead maps, similar to previously described methods (10).

Continuous variables are expressed as mean \pm SD or median and IQR. Two-sample $t$ tests or Wilcoxon rank sum tests were used to assess the differences in baseline (preimplantation) variables and change (postimplantation minus preimplantation) variables between scar burden groups and responders versus nonresponders. Categoric variables are expressed as number and percentage, and differences in baseline variables across the groups were assessed using $\chi^{2}$ tests. Paired $t$ tests were used to assess the changes in preimplantation and postimplantation continuous variables in the overall sample. Only the 157 patients with available LV lead position were included in analyses related to LV lead position. Logistic regression was performed to identify predictors of response to CRT. Because each patient may have multiple scar locations, 4 separate models were used to assess the 4 separate locations (lateral, posterior, anterior, and septal). For each model, the scar location was fit as a 3-level variable-no scarring, scarring at a nonlateral location, and scarring at a lateral location - and was repeated for other locations. This approach permitted the inclusion of all patients in the model, with a focus on the comparison between lateral and nonlateral locations (and similarly for other locations) within the group of patients who had scarring. Survival estimates were calculated by the KaplanMeier method. A log rank test was used to compare survival between groups. Cox proportional hazards models were used to assess multiple predictors of survival. A 2-sided $P$ value of less than 0.05 was considered significant.

\section{RESULTS}

Baseline characteristics of the 213 consecutive patients are summarized in Table 1. Eighty-four percent of the study subjects were men, and most patients (73\%) had ICM. Approximately half of the patients had left bundle branch block (LBBB); the other half had right bundle branch block (5\%), nonspecific intraventricular conduction delay $(9 \%)$, or ventricular paced rhythm $(31 \%)$ on the baseline electrocardiogram. Eighty-two percent of patients were taking angiotensin II receptor blockers or angiotensin-converting enzyme inhibitors, and $89 \%$ were taking $\beta$-blockers.

Clinical and echocardiographic parameters before and after CRT implantation are summarized in Table 2. NYHA class, BNP level, LVEF, LVESD, LVEDD, and MR severity were significantly improved after CRT.

A total of 651 scar segments and $157 \mathrm{LV}$ lead positions were identified in the 213 patients (a median of 2 [IQR,

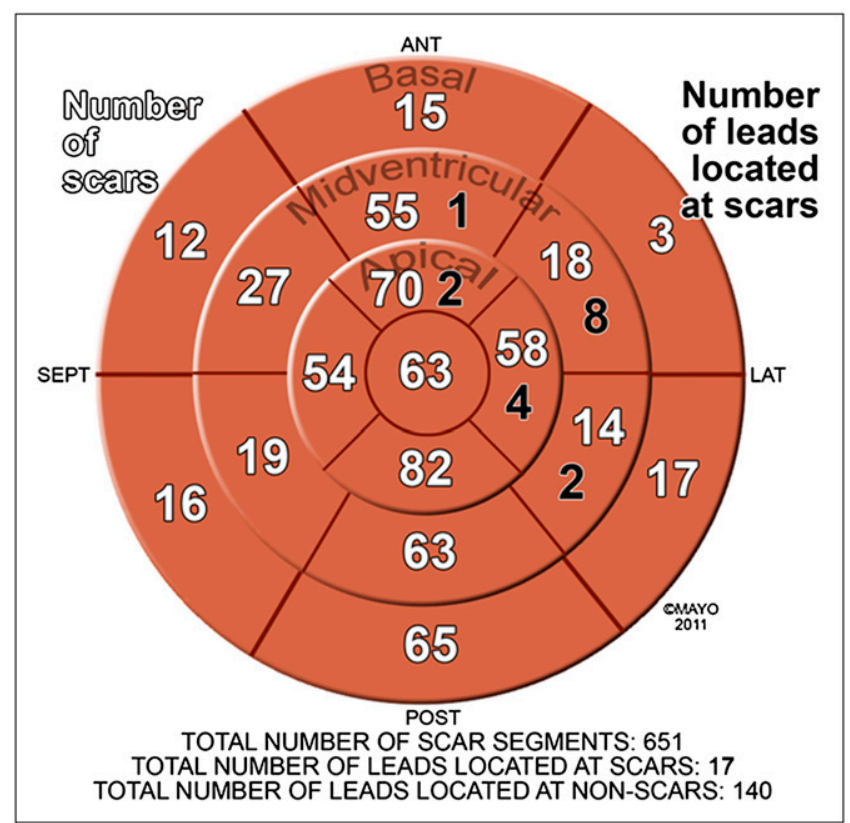

FIGURE 1. Distribution of scar sites and LV leads located in scar segment in 17-segment polar map. White number indicates number of leads in nonscarred segment in each location, and black number indicates number of LV leads located in scarred segment in each location. ANT $=$ anterior; LAT $=$ lateral; POST $=$ posterior; SEPT $=$ septal. 
TABLE 3

Univariate Logistic Models for CRT Response

\begin{tabular}{|c|c|c|c|}
\hline Variable & OR & $95 \% \mathrm{Cl}$ & $P$ \\
\hline Female vs. male sex & 0.93 & $0.39-2.21$ & 0.865 \\
\hline Age (per $10 \mathrm{y})$ & 1.01 & $0.73-1.40$ & 0.943 \\
\hline NICM vs. ICM & 1.59 & $0.79-3.20$ & 0.189 \\
\hline Chronic atrial fibrillation & 0.77 & $0.39-1.51$ & 0.444 \\
\hline NYHA class & 0.86 & $0.43-1.74$ & 0.683 \\
\hline LVEF (per 10\%) & 0.78 & $0.51-1.19$ & 0.251 \\
\hline QRS duration (per $10 \mathrm{~ms}$ ) & 1.05 & $0.96-1.15$ & 0.314 \\
\hline Left bundle branch block & 1.45 & $0.77-2.70$ & 0.247 \\
\hline LVEDD (per 10 mm) & 0.61 & $0.41-0.90$ & 0.012 \\
\hline LVESD (per 10 mm) & 0.71 & $0.45-1.13$ & 0.146 \\
\hline Right ventricular size & 0.79 & $0.56-1.12$ & 0.185 \\
\hline Right ventricular dysfunction & 0.94 & $0.67-1.32$ & 0.721 \\
\hline $\mathrm{MR}$ & 0.70 & $0.46-1.07$ & 0.103 \\
\hline Pulmonary artery systolic pressure (per 10 mm Hg) & 0.85 & $0.68-1.05$ & 0.128 \\
\hline Biventricular pacing, $99 \%-100 \%$ vs. $<99 \%$ & 1.45 & $0.75-2.83$ & 0.271 \\
\hline Lateral LV lead location & 1.45 & $0.65-3.24$ & 0.368 \\
\hline Number of viable myocardial segments & 1.04 & $1.01-1.06$ & 0.009 \\
\hline Number of scar segments & 0.84 & $0.76-0.94$ & 0.001 \\
\hline Extent of scarring (\% LV mass) & 0.97 & $0.95-0.99$ & 0.001 \\
\hline Lateral scar location & & & 0.049 \\
\hline No scar vs. nonlateral scar & 1.62 & $0.72-3.65$ & 0.240 \\
\hline Lateral scar vs. nonlateral scar & 0.63 & $0.29-1.36$ & 0.241 \\
\hline Posterior scar location & & & 0.004 \\
\hline No scar vs. nonposterior scar & 0.21 & $0.03-1.78$ & 0.153 \\
\hline Posterior scar vs. nonposterior scar & 0.08 & $0.01-0.67$ & 0.020 \\
\hline Anterior scar location & & & 0.060 \\
\hline No scar vs. nonanterior scar & 1.69 & $0.75-3.81$ & 0.206 \\
\hline Anterior scar vs. nonanterior scar & 0.68 & $0.31-1.47$ & 0.327 \\
\hline Septal scar location & & & 0.022 \\
\hline No scar vs. nonseptal scar & 1.54 & $0.71-3.31$ & 0.274 \\
\hline Septal scar vs. nonseptal scar & 0.50 & $0.23-1.09$ & 0.081 \\
\hline LV lead in scar segment & 0.94 & $0.34-2.61$ & 0.902 \\
\hline
\end{tabular}

0-6] scar segments per patient). Most (132/157; 84.1\%) LV leads were located at the lateral wall, and most $(83.1 \%)$ scar segments were distributed in the septal, anterior, posterior, and apical regions. Only $11 \%$ of LV leads were positioned in a scar segment (Fig. 1). In subjects who had LV lead data and at least 1 scar, scarring was present in more than $1 \mathrm{LV}$ segment in the majority (88\%), whereas the remaining $12 \%$ of patients had a single segment with scarring.

Using the predefined echocardiographic criteria, we identified $55.8 \%$ of patients as responders to CRT. Notably, the median number of viable segments was significantly higher in responders than in nonresponders ( 55 vs. 48), and the median total number of scar segments ( 1 vs. 3 ) and the median extent of scarring (6.0 vs. 18.0) were significantly lower in responders than in nonresponders. The number of patients with the LV lead in a scar segment was similar among responders and nonresponders (9 vs. 8). Univariate logistic regression models predicting CRT response are shown in Table 3. Larger preimplantation LVEDD (odds ratio $[\mathrm{OR}], 0.61 ; 95 \%$ confidence interval $[\mathrm{CI}], 0.41-0.90$; $P=0.01)$, greater number of scars (OR, 0.84; CI, $0.76-0.94 ; P=0.001$ ), and larger total scar burden (OR, 0.97 ; CI, 0.95-0.99; $P=0.001$ ) were associated with lower odds of CRT response. A greater number of viable myocardial segments was associated with higher odds of CRT response (OR, 1.04; CI, 1.01-1.06; $P=0.009$ ). There was evidence that CRT response differed by scar location. Patients with posterior scarring had lower odds of CRT response than those without posterior scarring (OR, 0.08; CI, 0.01-0.67; $P=0.020$ ). Patients with septal scarring, compared with those without septal scarring, had a trend for less CRT response (OR, 0.50; CI, 0.23-1.09; $P=0.081$ ). Only extent of scar burden remained significant in a multivariate model.

By logistical regression, scar extent of $22 \%$ LV mass was identified as the optimal cutoff point to predict response to CRT (sensitivity, 78.0\%; specificity, 47.9\%; OR, 0.97; CI, $0.95-0.99 ; P=0.001)$. Sixty-four percent of patients had scar extent $>22 \%$ using this threshold. Greater scar burden was present in patients who were significantly younger and who more often had ICM, lower LVEF, higher LVEDD, higher pulmonary artery systolic pressure, and more severe MR (Table 4). After CRT, patients with less scar burden had a greater improvement in LVEF. Other parameters were comparable between the 2 groups.

When patients were further dichotomized by the mean age of $70 \mathrm{y}$, the younger age group ( $\leq 70 \mathrm{y})$ with a scar 
TABLE 4

Comparison of Patients on Basis of Scar Burden

\begin{tabular}{|c|c|c|c|}
\hline Variable & Scarring extent $<22 \%$ & Scarring extent $\geq 22 \%$ & $P$ \\
\hline Age $(y)$ & $71.6 \pm 9.5$ & $67.9 \pm 8.9$ & 0.007 \\
\hline Male sex & $116(85)$ & $61(84)$ & 0.83 \\
\hline NICM & $48(35)$ & $7(10)$ & $<0.001$ \\
\hline Chronic atrial fibrillation & $38(28)$ & $20(27)$ & 0.96 \\
\hline NYHA class & $3.0 \pm 0.5$ & $3.0 \pm 0.4$ & 0.55 \\
\hline LVEF (before implantation), \% & $24.5 \pm 7.0$ & $22.1 \pm 7.0$ & 0.02 \\
\hline LVEDD (before implantation), $\mathrm{mm}$ & $63.3 \pm 8.3$ & $68.1 \pm 8.7$ & $<0.001$ \\
\hline LVESD (before implantation), $\mathrm{mm}$ & $55.7 \pm 8.5$ & $60.2 \pm 8.7$ & 0.004 \\
\hline Right ventricular size, grade 0-3 & $0.8 \pm 0.9$ & $1.0 \pm 0.9$ & 0.22 \\
\hline MR, grade $0-3$ & $1.4 \pm 0.8$ & $1.7 \pm 0.8$ & 0.02 \\
\hline Pulmonary artery systolic pressure (before implantation) (mm Hg) & $43.7 \pm 13.4$ & $52.9 \pm 15.9$ & $<0.001$ \\
\hline Change in NYHA class & $-0.7 \pm 0.9$ & $-0.5 \pm 0.8$ & 0.13 \\
\hline Change in QRS duration (ms) & $-9.3 \pm 39.1$ & $4.3 \pm 38.0$ & 0.04 \\
\hline Change in LVEF (\%) & $9.9 \pm 11.9$ & $2.8 \pm 6.7$ & $<0.001$ \\
\hline Change in LVEDD (mm) & $-2.9 \pm 7.9$ & $-1.8 \pm 3.5$ & 0.33 \\
\hline Change in LVESD (mm) & $-4.5 \pm 9.5$ & $-2.9 \pm 5.0$ & 0.39 \\
\hline Change in right ventricular size, grade $0-3$ & $-0.1 \pm 0.7$ & $-0.1 \pm 0.9$ & 0.82 \\
\hline Change in MR severity, grade $0-3$ & $-0.3 \pm 0.6$ & $-0.2 \pm 0.6$ & 0.35 \\
\hline Change in pulmonary artery systolic pressure $(\mathrm{mm} \mathrm{Hg})$ & $-4.7 \pm 13.2$ & $-5.7 \pm 15.3$ & 0.69 \\
\hline
\end{tabular}

burden $\leq 22 \%$ had a greater improvement in LVEF $(10.1 \% \pm 10.5 \%$ vs. $0.8 \% \pm 6.1 \% ; P<0.001)$, NYHA class $(-0.9 \pm 0.7$ vs. $-0.5 \pm 0.8 ; P=0.02)$, and LVESD $(-6.3 \pm 7.0$ vs. $-1.1 \pm 4.1 ; P=0.009)$ than the younger group with greater scar burden. However, in the older age group $(>70 \mathrm{y}$ ), improvements in LVEF, NYHA class, LVESD, and LVEDD were similar between those with greater and lesser scar burden.

Univariate and multivariate survival analyses using Cox proportional hazards models are shown in Table 5. The total number of deaths in the study group was 69. The multivariate model showed that older age (hazard ratio [HR], 1.36; 95\% CI, 1.01-1.83; $P=0.05)$ and more severe pre-CRT MR (HR, 1.72; 95\% CI, 1.25-2.37; $P<0.001)$ were independently associated with higher risk of death, whereas nonischemic cardiomyopathy (NICM) (HR, 0.40; 95\% CI, $0.18-0.89 ; P=0.03$ ), LBBB (HR, 0.58; 95\% CI, 0.34$0.98 ; P=0.04$ ), and lateral LV lead location (HR, 0.52; 95\% CI, 0.30-0.91; $P=0.02$ ) were independently associated with better survival. Scar extent and location were not univariate predictors of survival. When patients were substratified by age $(\leq 70$ y or $>70 y)$, Kaplan-Meier estimates showed that younger patients with scar burden $>22 \%$ had significantly worse survival than those with less scar burden (Fig. 2A), whereas older patients had similar survival between the 2 scar burden groups (Fig. 2B).

\section{DISCUSSION}

To our knowledge, this is the largest study to date to address the relationships between scar burden, scar location, and LV lead position in CRT. The study revealed 3 main findings: most CRT LV leads were positioned in the LV lateral wall, where scars were infrequently present by radionuclide MPI in this cohort; greater total scar burden was significantly associated with a lack of response to CRT; and less total scar burden was associated with greater survival but only in younger CRT recipients.

The primary objective of CRT is to improve LV contractility and efficiency via an additional lead in the LV free wall to achieve biventricular resynchronization. Therefore, the LV wall substrate and its interaction with the LV lead may affect CRT outcomes. Several studies have examined the relationships between myocardial scarring, LV lead position, and CRT response, but cohorts have been small and the relationships not fully characterized. Ypenburg et al. reported that $29.4 \%$ of their patients had transmural scarring in the region of the LV lead. In these patients, NYHA class, 6-min walking distance, quality-of-life score, LVEF, and LV dimensions did not improve from baseline to follow-up (10). However, the Ypenburg study had a higher percentage of patients with scarring located in the LV lead region than did the current study, which had only $11 \%$ of LV leads in a scar segment and no significant difference in LV lead position in scar segment between nonresponders and responders. The earlier study also had only 51 patients, compared with our current report of 213 patients. Notably, the prior study reported that the inverse relationship between scar burden and CRT response was maintained when patients with scar tissue in the LV lead position were excluded (10). Other previous cardiac magnetic resonance (CMR; $n=40$ patients) and nuclear imaging ( $n=50$ patient) studies have addressed the relationships between scar location, LV lead position, and CRT response $(11,23)$. The contrast- 
TABLE 5

Univariate and Multivariate Predictors of All-Cause Mortality

\begin{tabular}{|c|c|c|c|c|c|c|}
\hline \multirow[b]{2}{*}{ Variable } & \multicolumn{3}{|c|}{ Univariate analysis } & \multicolumn{3}{|c|}{ Multivariate analysis } \\
\hline & $\mathrm{HR}$ & $95 \% \mathrm{Cl}$ & $P$ & $\mathrm{HR}$ & $95 \% \mathrm{Cl}$ & $P$ \\
\hline Female vs. male sex & 0.62 & $0.28-1.35$ & 0.224 & & & \\
\hline Age (per 10 y) & 1.56 & $1.17-2.08$ & 0.003 & 1.36 & $1.01-1.83$ & 0.046 \\
\hline NICM vs. ICM & 0.41 & $0.19-0.85$ & 0.017 & 0.40 & $0.18-0.89$ & 0.025 \\
\hline Chronic atrial fibrillation & 1.56 & $0.95-2.55$ & 0.079 & & & \\
\hline NYHA class & 1.80 & $1.09-2.98$ & 0.022 & & & \\
\hline LVEF (per 10\%) & 1.15 & $0.81-1.62$ & 0.429 & & & \\
\hline QRS duration (per $10 \mathrm{~ms}$ ) & 1.01 & $0.94-1.08$ & 0.881 & & & \\
\hline LBBB & 0.60 & $0.37-0.98$ & 0.041 & 0.58 & $0.34-0.98$ & 0.040 \\
\hline LVEDD (per $10 \mathrm{~mm}$ ) & 0.94 & $0.72-1.23$ & 0.673 & & & \\
\hline LVESD (per 10 mm) & 0.93 & $0.65-1.35$ & 0.714 & & & \\
\hline Right ventricular size & 1.31 & $1.02-1.69$ & 0.036 & & & \\
\hline Right ventricular dysfunction & 1.29 & $1.01-1.66$ & 0.044 & & & \\
\hline MR & 1.75 & $1.28-2.40$ & $<0.001$ & 1.72 & $1.25-2.37$ & $<0.001$ \\
\hline Pulmonary artery systolic pressure (per $10 \mathrm{~mm} \mathrm{Hg}$ ) & 1.22 & $1.05-1.42$ & 0.011 & & & \\
\hline Biventricular pacing, $99 \%-100 \%$ vs. $<99 \%$ & 0.69 & $0.38-1.27$ & 0.238 & & & \\
\hline Lateral LV lead location & 0.47 & $0.28-0.80$ & 0.005 & 0.52 & $0.30-0.91$ & 0.021 \\
\hline Total scar number & 1.00 & $0.93-1.07$ & 0.966 & & & \\
\hline Extent of scarring (\% LV) & 1.00 & $0.99-1.01$ & 0.971 & & & \\
\hline Lateral scar location & & & 0.927 & & & \\
\hline No scar vs. nonlateral scar & 0.89 & $0.49-1.62$ & 0.700 & & & \\
\hline Lateral scar vs. nonlateral scar & 0.96 & $0.54-1.69$ & 0.886 & & & \\
\hline Posterior scar location & & & 0.670 & & & \\
\hline No scar vs. nonposterior scar & 1.27 & $0.48-3.38$ & 0.632 & & & \\
\hline Posterior scar vs. nonposterior scar & 1.46 & $0.58-3.70$ & 0.420 & & & \\
\hline Anterior scar location & & & 0.476 & & & \\
\hline No scar vs. nonanterior scar & 0.77 & $0.43-1.37$ & 0.367 & & & \\
\hline Anterior scar vs. nonanterior scar & 0.72 & $0.41-1.27$ & 0.252 & & & \\
\hline Septal scar location & & & 0.909 & & & \\
\hline No scar vs. nonseptal scar & 0.84 & $0.53-1.66$ & 0.825 & & & \\
\hline Septal scar vs. nonseptal scar & 1.07 & $0.61-1.90$ & 0.808 & & & \\
\hline
\end{tabular}

enhanced cardiac MRI study focused mainly on patients with posterolateral scarring and the lack of clinical CRT response but did not address the contribution of total scar burden to this relationship (23). The prior nuclear imaging study, similar to ours, addressed both overall scar burden and LV lead position in scar segments and reported that higher overall scar burden and greater scar density near the LV lead tip were associated with an unfavorable CRT response (11). However, the overall contributions of scar burden and lead location were not defined, and both prior studies were smaller than the current study. Most patients with scarring in our study had multiple scars located in 2 or more LV areas. Scar segments were most often present in the anterior, septal, posterior, and apical regions, whereas the lateral wall, an optimal location for LV lead placement, was often spared. Hence, scar location did not appear to have a significant impact on CRT outcome, given the evidence of a mismatch between scar location and LV lead position.

The site of latest activation in relation to LV lead location is another important factor affecting CRT outcome. A recent report randomized 220 patients with advanced HF to targeted LV lead placement, guided by speckle-tracking echocardiography versus unguided lead placement and demonstrated that targeted lead placement resulted in a higher LVEF response rate than untargeted lead placement (70\% vs. 55\%; $P=0.03$ ) (24). Individualized resynchronization using the site of latest electrical or mechanical activation may, therefore, be more efficacious than universal placement of the LV lead in the lateral wall.

We also found that greater scar burden was significantly associated with lack of response to CRT. This finding supports a prior small study demonstrating that the extent of myocardial viability as measured by myocardial contrast echocardiography is more critical than scar location for predicting CRT outcomes (25). Similarly, Ypenburg et al. reported an association between the extent of viability measured by ${ }^{18} \mathrm{~F}$-FDG SPECT and the improvement in LVEF 6 mo after CRT (9). In that study, total scar burden was inversely related to the change in LVEF. In line with these observations, we found that patients with greater scar burden had more ICM etiology, shorter QRS duration, lower LVEF, and greater LV remodeling at baseline, implying attenuated benefits from CRT. Patients with greater scar burden had significantly less improvement in LVEF than patients with less scar burden. Notably, in our entire cohort, patients with greater scar burden were significantly younger 


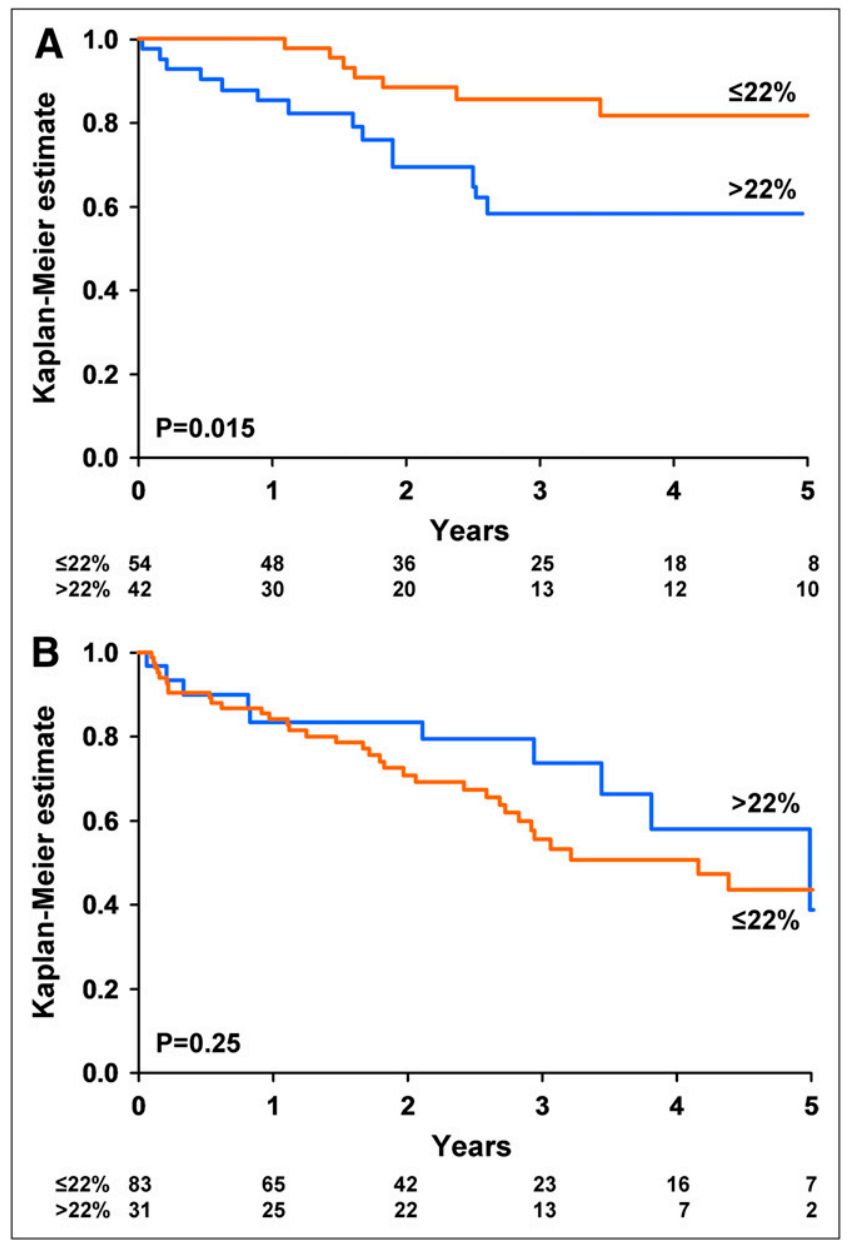

FIGURE 2. Kaplan-Meier analysis of the impact of scar burden on survival after cardiac resynchronization therapy: patients aged $\leq 70$ y (A) and patients $>70$ y (B).

than those with less scar burden. In the younger group, those with scar burden $\leq 22 \%$ had more significant improvement in LV function and NYHA class than those with scar burden $>22 \%$. However, in the older age group, these differences based on scar burden were not present.

Published data on scar burden predicting survival after CRT are scarce. Adelstein et al. reported that extensive scar burden in ICM patients unfavorably affected clinical, LV functional, and survival outcomes after CRT, regardless of baseline dyssynchrony measures (26). However, they detected scar burden only in ICM patients. In our study of 213 patients with $27 \%$ NICM, the impact of scar burden on survival after CRT paralleled the impact of scar burden on clinical benefits and LV reverse remodeling after CRT, especially in younger patients. These unique findings extend the potential value of scar burden in estimating survival benefits of CRT in both ICM and NICM and in the younger, compared with the older, age groups. In our older patients, less scar burden was not associated with a higher survival rate. A potential explanation is that concomitant comorbid conditions often seen in elderly patients may also affect survival. Swindle et al. pointed out that advanced age and elevated comorbidity score were independent predictors of in-hospital mortality after CRT or implantable cardioverter-defibrillator placement (27). Older patients may also have higher risk of noncardiac death; however, we did not differentiate the cause of death after CRT.

Several limitations of the current study exist. In the present study, SPECT or PET MPI was used to evaluate myocardial scarring or viable tissue. The choice of MPI was at the discretion of each clinician. Although differences exist, SPECT and PET perfusion tracers both require myocardial perfusion and viability for tracer uptake and are widely used clinically, allowing for future widespread larger studies and potential generalizability. However, it is widely accepted that PET metabolic imaging with ${ }^{18} \mathrm{~F}$-FDG may be more precise in differentiating scarring from viable myocardium. PET metabolic imaging with ${ }^{18} \mathrm{~F}-\mathrm{FDG}$ was not available in the current study. Future larger studies should consider inclusion of PET metabolic imaging.

In the current study, CRT implantation occurred from 2002 to 2008. Device technology has changed over this time period and may be a potential limitation in the application of findings to current CRT recipients. However, the basic concepts of CRT have remained constant over this period.

The study nature is retrospective. Only about one third of patients who underwent CRT implantation during the study period underwent radionuclide MPI. The referral bias associated with patients undergoing radionuclide MPI may also have an impact on study results. However, there were no significant differences in the cohort who underwent versus those who did not undergo radionuclide MPI, and the current cohort is similar to other published cohorts of CRT patients. The time between nuclear MPI and CRT implantation was $1 \mathrm{y}$, which is another limitation of this retrospective study.

The focus of the study was myocardial scarring burden in relation to LV lead position and CRT outcome. We did not measure the site of latest electrical and mechanical activation of the LV in relationship with LV lead location.

Although the entire cohort had survival follow-up, clinical follow-up is incomplete and whether death was cardiac or noncardiac was not known.

\section{CONCLUSION}

The probability of an LV lead being positioned in a scar segment is low in this cohort of patients, and LV lead position in scar location does not appear to have a significant impact on CRT response in this group. On the other hand, total scar burden is significantly lower in CRT responders than in nonresponders. Less scar burden is associated with a greater improvement in HF and survival but only in younger CRT recipients. Scar burden measured by nuclear imaging may be considered before CRT implantation, especially in patients younger than $70 \mathrm{y}$. Prospective multicenter studies should be conducted to 
determine whether this technique would help to improve CRT patient selection.

\section{DISCLOSURE STATEMENT}

The costs of publication of this article were defrayed in part by the payment of page charges. Therefore, and solely to indicate this fact, this article is hereby marked "advertisement" in accordance with 18 USC section 1734.

\section{ACKNOWLEDGMENTS}

We acknowledge Kelly Brooke and Tracy L. Webster for their contributions to data acquisition. Dr. Cha received research grants from Medtronic and St. Jude Medical, and Dr. Chareonthaitawee received a research grant from Astellas Pharma. No other potential conflict of interest relevant to this article was reported.

\section{REFERENCES}

1. Roger VL, Weston SA, Redfield MM, et al. Trends in heart failure incidence and survival in a community-based population. JAMA. 2004;292:344-350.

2. Abraham WT, Fisher WG, Smith AL, et al. Cardiac resynchronization in chronic heart failure. N Engl J Med. 2002;346:1845-1853.

3. Auricchio A, Stellbrink C, Sack S, et al. Long-term clinical effect of hemodynamically optimized cardiac resynchronization therapy in patients with heart failure and ventricular conduction delay. J Am Coll Cardiol. 2002;39: 2026-2033.

4. Bristow MR, Saxon LA, Boehmer J, et al. Cardiac-resynchronization therapy with or without an implantable defibrillator in advanced chronic heart failure. $N$ Engl J Med. 2004;350:2140-2150.

5. Cha YM, Rea RF, Wang M, et al. Response to cardiac resynchronization therapy predicts survival in heart failure: a single-center experience. J Cardiovasc Electrophysiol. 2007; 18:1015-1019.

6. Bax JJ, Marwick TH, Molhoek SG, et al. Left ventricular dyssynchrony predicts benefit of cardiac resynchronization therapy in patients with end-stage heart failure before pacemaker implantation. Am J Cardiol. 2003;92:1238-1240.

7. Bax JJ, Abraham T, Barold SS, et al. Cardiac resynchronization therapy: part 1issues before device implantation. J Am Coll Cardiol. 2005;46:2153-2167.

8. Aggarwal NR, Martinez MW, Gersh BJ, Chareonthaitawee P. Role of cardiac MRI and nuclear imaging in cardiac resynchronization therapy. Nat Rev Cardiol. 2009;6:759-770.

9. Ypenburg C, Schalij MJ, Bleeker GB, et al. Extent of viability to predict response to cardiac resynchronization therapy in ischemic heart failure patients. J Nucl Med. 2006;47:1565-1570.

10. Ypenburg C, Schalij MJ, Bleeker GB, et al. Impact of viability and scar tissue on response to cardiac resynchronization therapy in ischaemic heart failure patients. Eur Heart J. 2007;28:33-41.

11. Adelstein EC, Saba S. Scar burden by myocardial perfusion imaging predicts echocardiographic response to cardiac resynchronization therapy in ischemic cardiomyopathy. Am Heart J. 2007;153:105-112.
12. Sciagrà R, Giaccardi M, Porciani MC, et al. Myocardial perfusion imaging using gated SPECT in heart failure patients undergoing cardiac resynchronization therapy. J Nucl Med. 2004;45:164-168.

13. Kennedy JW, Killip T, Fisher LD, Alderman EL, Gillespie MJ, Mock MB. The clinical spectrum of coronary artery disease and its surgical and medical management, 1974-1979. The Coronary Artery Surgery study. Circulation. 1982;66: III16-III23.

14. Logeart D, Thabut G, Jourdain P, et al. Predischarge B-type natriuretic peptide assay for identifying patients at high risk of re-admission after decompensated heart failure. J Am Coll Cardiol. 2004;43:635-641.

15. Miller TD, Rajagopalan N, Hodge DO, Frye RL, Gibbons RJ. Yield of stress single-photon emission computed tomography in asymptomatic patients with diabetes. Am Heart J. 2004;147:890-896.

16. Rajagopalan N, Miller TD, Hodge DO, Frye RL, Gibbons RJ. Identifying highrisk asymptomatic diabetic patients who are candidates for screening stress single-photon emission computed tomography imaging. J Am Coll Cardiol. 2005;45:43-49.

17. Cerqueira MD, Weissman NJ, Dilsizian V, et al. Standardized myocardial segmentation and nomenclature for tomographic imaging of the heart: a statement for healthcare professionals from the Cardiac Imaging Committee of the Council on Clinical Cardiology of the American Heart Association. Circulation. 2002;105:539-542.

18. Khandaker MH, Miller TD, Chareonthaitawee P, Askew JW, Hodge DO, Gibbons RJ. Stress single photon emission computed tomography for detection of coronary artery disease and risk stratification of asymptomatic patients at moderate risk. J Nucl Cardiol. 2009;16:516-523.

19. Knuuti J, Schelbert HR, Bax JJ. The need for standardisation of cardiac FDG PET imaging in the evaluation of myocardial viability in patients with chronic ischaemic left ventricular dysfunction. Eur J Nucl Med Mol Imaging. 2002;29:1257-1266.

20. Bleeker GB, Bax JJ, Fung JW-H, et al. Clinical versus echocardiographic parameters to assess response to cardiac resynchronization therapy. Am J Cardiol. 2006;97:260-263.

21. Wokhlu A, Rea RF, Asirvatham SJ, et al. Upgrade and de novo cardiac resynchronization therapy: impact of paced or intrinsic QRS morphology on outcomes and survival. Heart Rhythm. 2009;6:1439-1447.

22. Riedlbauchová L, Brunken R, Jaber WA, et al. The impact of myocardial viability on the clinical outcome of cardiac resynchronization therapy. J Cardiovasc Electrophysiol. 2009;20:50-57.

23. Bleeker GB, Kaandorp TA, Lamb HJ, et al. Effect of posterolateral scar tissue on clinical and echocardiographic improvement after cardiac resynchronization therapy. Circulation. 2006;113:969-976.

24. Khan FZ, Virdee MS, Begley D, et al. Targeted left ventricular lead placement using speckle tracking echocardiography improves the acute hemodynamic response to cardiac resynchronization therapy: a randomized controlled trial. $J \mathrm{Am}$ Coll Cardiol. 2011;57:E2033.

25. Hummel JP, Lindner JR, Belcik JT, et al. Extent of myocardial viability predicts response to biventricular pacing in ischemic cardiomyopathy. Heart Rhythm. 2005;2:1211-1217.

26. Adelstein EC, Tanaka H, Soman P, et al. Impact of scar burden by single-photon emission computed tomography myocardial perfusion imaging on patient outcomes following cardiac resynchronization therapy. Eur Heart J. 2011;32:93103.

27. Swindle JP, Rich MW, McCann P, Burroughs TE, Hauptman PJ. Implantable cardiac device procedures in older patients: use and in-hospital outcomes. Arch Intern Med. 2010;170:631-637. 